\title{
Time to think
}

Our intuitive ideas about the world - the wisdom we often call 'common sense' hasn't stood up well to the tests of science. Many things that were once obvious have turned out to be wrong. The Earth isn't at the centre of the Universe. The world around us, when we get down to its quantum behaviour, doesn't even have properties that are independent of our observation of them.

In human sciences, such as psychology, things are much the same. It wasn't long ago that philosophers put man on a pedestal as the one creature able to act consistently through reason and conscious action. Yet numerous experiments over the past few decades have clearly established that much of that conscious control is actually a comforting illusion; we frequently act on unconscious stimuli, and our conscious brain fills in a convincing rationalization afterwards (for example, see J. Bargh, Perspect. Psychol Sci. 3, 73-79; 2008).

It's just not an intuitive world, after all. But is common sense, then, completely useless? Or does it possibly still hold some valuable clues for science? In a recent paper, physicist Lee Smolin makes a convincing case for the latter, especially when it comes to the understanding the tricky nature of time (preprint at http://arXiv.org/abs/1310.8539; 2013) and cosmology as a whole. Our natural intuition about time may tell us something profound about the nature of the Universe.

As conscious individuals, our direct experience makes us feel that the past is obviously and inherently different from the future. The past has happened and left a record; we can discuss it and, within limits, agree on some facts about what happened. The past is definite, unlike the future. Indeed, we feel the passage of time as real; it moves us perpetually from uncertainties into certainties. We have no choice about whether or not to take part in this movement.

Our common sense, in other words, tells us that time has an arrow. And yet much of today's physics insists the opposite - that this arrow is only illusion. In particular, there is no preferred direction of time in the classical dynamics of Newton, or in more advanced theories such as general relativity or quantum theory, which share the same basic structure. Starting with Boltzmann in the 19th century, many physicists have laboured to demonstrate that such microreversibility can lead to an effective direction of time for human

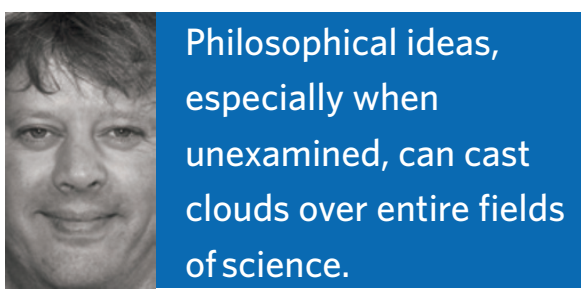

experience; yet such efforts still ultimately trace time's irreversibility back to the very low entropy initial condition of the Universe just after the Big Bang (R. Penrose, The Emperor's New Mind; Penguin, 1991). The basic laws themselves remain timeless.

Taking this idea seriously, many cosmologists today propose that our experience of time really is a kind of illusion. It's easy enough, if you mingle space and time inextricably together as relativity does, to see the Universe as one timeless whole situated in a higher dimensional setting. In this view - Smolin refers to it as "timeless naturalism" - we're subjectively aware of time only because we're stuck in it, localized in the time dimension, and moving along it, only ever aware of the present moment.

Mathematically, okay. But examine the idea more closely, Smolin suggests, and the price for accepting it seems quite high. It leaves many things completely unexplained. After all, if the world has no time, then why do we experience it that way? And why as a set of moments? Some philosophers refer to this as the 'hard' problem of time, and it's only a beginning. Why, Smolin asks, does time seem to us to have a one-dimensional character rather than something else? Relativity does not demand this.

Finally, why do we remember the past but not the future? Timeless naturalism has no answer, as it doesn't even distinguish between future and past. The Universe, in that view, is a single inseparable whole, a block of spacetime. But we most certainly do not experience it that way.

In contrast, Smolin points out, these problems mostly evaporate if we accept the common sense notion of time as a real thing, passing us by, and having a direction. Call this 'temporal naturalism'. This view immediately solves the hard problem of time, as it suggests that we experience the world as a linear succession of moments precisely because that's how the world really is. Likewise, it explains why we remember past but not future: the past actually happened and, indeed, caused the present.
We don't remember the future because it is not yet real.

That's philosophically satisfying, but Smolin also suggests that this view has major advantages in a more specific scientific sense by altering the kinds of questions we think worthy of further research. In particular, it opens the door to exploring the possibility that the fundamental laws of physics may actually be time asymmetric.

The perspective of timeless naturalism, he notes, more or less forecloses consideration of several fundamental issues. If the Universe is timeless, then there's no real history and no sense in asking how the laws of physics as we know them came to be that way. But perhaps they did undergo evolution at some stage. In a timeless Universe, one also takes the initial condition of the Universe as a given, not as a consequence of anything. Yet asking why the Universe had the very special low-entropy state it seems to have had might also be a profitable line of inquiry. It does indeed seem strange that our experience of time could reflect facts about the origins of the Universe, but that's what logic tells us. Hence, we may only understand time in a deep way if we can learn how and why the early Universe came to be configured in such a special way.

Smolin's argument overall has a strongly philosophical character, teasing out logical relations and meanings, criticizing different positions on how to interpret the reality around us. This retreat into philosophy, in part, reflects a difficulty confronting cosmological theorizing in the absence of easy experimental data to constrain ideas. But his argument emphasizes how philosophical ideas, especially when unexamined, can cast clouds over entire fields of science, influencing what seems interesting or not.

"It is mostly the unconscious reliance on the metaphysical fantasies behind timeless naturalism", he claims, "that is responsible for current crises in cosmological theorizing."

In trying to move beyond this crisis, Smolin argues that the initial state of the universe might be linked to some evolutionary process that governs the creation of new universes from old during black-hole formation. It sounds a little fantastic, but it's a specific idea worth exploring and testing. Some idea along these lines might restore time to its rightful place - as something very much more than an illusion.

\section{MARK BUCHANAN}

